\title{
Improving the Social Capital of Trust-based Competitive Multi-Agent Systems by Introducing Meritocracy
}

\author{
Antonello Comi, Lidia Fotia and Domenico Rosaci \\ University of Reggio Calabria, DIIES Department \\ \{antonello.comi,lidia.fotia,domenico.rosaci\}@unirc.it
}

\begin{abstract}
A main issue in competitive Multi-Agent Systems is that of allowing self-interested agents to mutually cooperate in those cases where the local agent resources are not sufficient to satisfy user requests. To this end, it is necessary to introduce an internal organization for allowing agents to form suitable friendships and groups facilitating the collaboration. In the past, several approaches have been proposed aiming at forming agent coalitions which maximize the profit of the group or the individual agent. However, this viewpoint could introduce some negative side-effects, namely (i) it can lead to reward the most aggressive agents, also if they have bad social behaviours or (ii) it could introduce a sort of social flattening, without taking into account the differences among the agents in terms of merit. To face this issue, in this paper we propose an algorithm for forming friendships and groups which, instead of maximizing individual or global profit, tries to optimize a social capital represented by the mutual trust relationships.

We theoretically prove that the application of our algorithm leads the competition to reward those agents exhibiting the most virtuous behaviours, introducing meritocracy in the system, not only rewarding effective agent performances but also encouraging correct behaviours.
\end{abstract}

\section{INTRODUCTION}

A COMPETITIVE Multi-Agent System (MAS) can be viewed as a virtual community composed of selfinterested agents that often need to interact with each other to accomplish complex tasks. Although the introduction of cooperative behaviours seem necessary for obtaining better results, it is not possible for agents to assume that the others will cooperate sincerely, since agents are not interested in global outcome but only their own [4], [1]. For this reason, maybe some of these interactions will be done by some agent with the intention of deceiving its interlocutor, which is also a competitor. Therefore, in these systems, an agent has the problem of accurately selecting its partner, since both incomes and outcomes depend on this choice, and trust-based approaches are a possible solution to face this problem.

The issue of defining and measuring the trust between members of a community has been actively faced in the Social Sciences research field, and a common belief of most of the existing studies is that the concept of trust is strictly linked to the concept of social capital [15]. Social capital is

This work has been partially supported by the TENACE PRIN Project ( $\mathrm{n}$. 20103P34XC) funded by the Italian Ministry of Education, University and Research. a broad concept referring to the collective value associated with a community of individuals, and has been defined as "the density of interactions that is beneficial to the members of a community" [20].

Social capital is widely acknowledged as the most important asset of any social community and, more in general, of any organization. Therefore, the task of measuring trust is of the utmost relevance to understand the benefits a user can draw from her/his membership to a particular social network.

In our scenario, we suppose that the cooperation among agents can be improved by introducing a social organization in the agent community. More in particular, such an organization is realized by allowing that an agent can have some friends, collected in a friendship set, and moreover he can join with some groups, with the advantage that a collaboration requested to a friend or a member of a common group is obtained for free.

In this scenario the main question is: which is the best organization for the agent community? In our vision, we assume that the best organization is that maximizing the social capital, that we will define as the average advantage, in terms of trust, that the whole community obtains from that organization.

In the past, several approaches [9], [5], [19] have been proposed aiming at forming agent coalitions which maximize the utility of the group or the individual agent. In other words, these approaches have the purpose of optimizing some measure of profit. However, this viewpoint could introduce some negative side-effects. In particular, if the approach is based on maximizing the local profit, it can lead to reward the most aggressive agents, also if they have bad social behaviours (e.g. misleading or fraudolent agents). Moreover, if the approach tries to optimize a global profit, it could introduce a sort of social flattening, without taking into account the differences among the agents in terms of merit.

To face this issue, in our approach we propose an algorithm for forming friendships and groups which, instead of maximizing individual or global profit, tries to optimize a social capital represented by the mutual trust relationships.

In particular, we will provide two main contributions. First, we theoretically prove that our algorithm leads to continuously increase the social capital of the community. Then, enough interestingly, we also prove that the application of our algo- 
rithm leads the competition to reward those agents exhibiting the most virtuous behaviours, i.e. those that the community perceives as the most competent and honest. In other words, our approach introduces meritocracy in the system, not only rewarding effective agent performances but also encouraging correct behaviours.

The plan of the paper is as follows. In Section II we describe a reference scenario for competitive agents. Then, in Section III we introduce our trust model while Section IV present the FGF algorithm we propose for forming friendships and groups. Next, in Section V we provide two important results related to FGF algorithm. In Section VI we discuss some related work and, finally in Section VII we draw our conclusions and discuss our ongoing research.

\section{THE REFERENCE SCENARIO}

Let $U$ be a set of users and let $A$ be a set of agents competing for satisfying the users' requests. In particular, each user $u \in U$ can submit to an agent $a \in A$ a service request $r_{\gamma}$ falling in the category $\gamma \in C$, where $C$ is a set of pre-defined categories. The user $u$ will pay a fixed price $p$ to the agent $a$, after the service has been provided.

Each agent $a$ is provided with an expertise $e_{a}(\gamma)$ for each category $\gamma \in C$, where the expertise represents the agent's capability to correctly providing a service falling in $\gamma$. The expertise $e_{a}(\gamma)$ is a value ranging in the interval [0..1], where $e_{a}(\gamma)=1$ (resp. $e_{a}(\gamma)=0$ ) means that $a$ provides services falling in $\gamma$ with the maximum (resp. minimum) quality of service. When the service has been provided, the user $u$ returns a feedback $f$ to $a$, where $f$ is a value falling in [0..1], such that $f=0$ (resp. $f=1$ ) represents the minimum (resp. maximum) satisfaction perceived by $u$ for the service provided by $a$.

Each agent $a$ has a set of friend agents, denoted by $F_{a}$, where $F_{a} \subseteq A$. Moreover, some groups of agents can be formed in this scenario. Let $G$ be the set of these groups, where each group $g \in G$ is a set of agents, such that $g \subseteq A$. The names of agents and groups are registered in a Directory Facilitator $(D F)$, as it is usual in multi-agent systems. The $D F$ also provides a mapping agents $(g)$ receiving as input a group $g \in G$ and returning the set of the agents which are members of $g$.

In order to satisfy a service request concerning the category $\gamma$, the agent $a$ can request the contribution of another user $b$, that can accept or refuse the contribution request. If $b$ accepts, a price $c p$ must be paid by $a$ to $b$ after the contribution has been provided by $b$. However, if $b$ is a friend of $a$ or $a$ and $b$ are in the same group, i.e. $b \in F_{a} \bigvee \exists g \in G: a, b \in g$, then $b$ will accept the request and will provide the contribution for free.

In our scenario, we suppose that an agent can request a maximum number of $c M a x$ contributions.

Moreover, in order to select those agents that are the best candidates for requesting contribution, the agent $a$ can require the opinion of the other agents. In particular, $a$ can send to an agent $c$ a request for obtaining a recommendation $\operatorname{rec}_{b}(\gamma)$ about the expertise $e_{b}(\gamma)$ of $b$ in a given category $\gamma$. In other words, $\operatorname{rec}_{b}(\gamma)$ is an estimation of $e_{b}(\gamma)$ provided by $c$. The recommendation request can be accepted or refused by $c$, and in the case of accepting, a price $r p$ is paid by $a$ to $c$. However, if $c$ is a friend of $a$ or $a$ and $c$ are in the same group, i.e. $c \in$ $F_{a} \bigvee \exists g \in G: a, c \in g$, then $c$ will accept the recommendation request and will provide the recommendation for free.

In our scenario, we suppose that an agent can request a maximum number of $r$ Max recommendations.

\section{TRUST MEASURES: RELIABILITY, REPUTATION AND HONESTY}

We assume that in the scenario described above it is possible that misbehaviours are adopted by some agent. In particular, an agent $b$ that has been requested for a contribution by $a$ could provide a service with a quality lesser than that corresponding to his actual expertise. Analogously, an agent $c$ that has been requested for a recommendation by $a$ about the agent $b$ could indicate a value for the expertise of $b$ different from the value that $c$ really knows.

In order to manage these possible misbehaviours, the agent $a$ associates three trust values to each agent $b$ with which he interacted in the past. The first value, denoted by $\operatorname{rel}(b, \gamma)$ is called reliability of $b$ with respect to the category $\gamma$, and represents how much $a$ trusts in the capability of obtaining good contribution from $b$ in service falling in category $\gamma$.

When $a$ receives from a user a feedback $f$ for a service, he computes for each agent $b$ which provided a contribution for that service, the component of the feedback associated with $b$. This component $f_{b}$ will be obtained by considering the feedback $f$ and possible additional information $i_{b}$ that $a$ has about the actual contribution provided by $b$. Formally, we define a mapping $h$ such that $f_{b}=h\left(f, i_{b}\right)$. For example, assume that the service provided by $a$ is represented by an integer value $p$. The user $u$ when receiving $p$ as the response to his request, will provide the feedback $f$ as the percentage error with respect to the correct answer $r$. Then, in this case, $f_{b}$ can be computed as the percentage error associated with the contribution $r_{b}$ provided by $b$ with respect to the correct answer $r$. In the case we do not have any additional information $i_{b}$, we assume that each feedback component $f_{b}=f$. In other words, in this case the agent $a$ entirely transfers the responsibility of his feedbacks to his contributors. The reliability $\operatorname{rel}(b, \gamma)$ is defined as the arithmetical mean of all the feedback components associated with $b$, related to feedbacks that $a$ received from users for services falling in the category $\gamma$.

The second trust value, denoted by hon $(b)$, is called honesty of $b$ when providing a recommendation. This value is computed by considering, for all the $l$ feedback components $f_{c}^{1}, \ldots, f_{c}^{l}$ that $a$ received from users and that are related to services which have been provided by a contributor $c$ recommended by $b$, the percentage difference between $f_{c}^{l}$ and the recommendation $r_{l}$ provided by $b$ and related to $c$ (i.e. we compute dif $\left.f_{l}=\frac{\left|r_{l}-f_{b}^{l}\right|}{r_{l}}\right)$. The value hon $(b)$ is defined as the arithmetical mean of all these $\operatorname{dif} f_{l}$ values. 
Finally, the third value, denoted by $\operatorname{rep}(b, \gamma)$, is called reputation of $b$ with respect to the category $\gamma$, and represents how much the agents interrogated by $a$ estimate the capability of $b$ in the category $\gamma$. The reputation $\operatorname{rep}(b, \gamma)$ is obtained as the weighted mean of all the recommendations that other agents provided to $a$ about $b$ in the category $\gamma$, where the weight of each recommendation provided by an agent $c$ is considered equal to hon $(c)$.

For all the agents $b$ that $a$ did not contact in the past for requesting contributions in category $\gamma, \operatorname{rel}(b, \gamma)$ is set equal to crel. Analogously, for all the agents $b$ that $a$ did not contact in the past for which $a$ did not receive recommendations in category $\gamma, \operatorname{rep}(b, \gamma)$ is set equal to $\operatorname{crep}$. Finally, for all the agents $b$ that $a$ did not contact for requesting recommendations in the past, hon (b) is set to chon. The values crel, crep and chon are cold start values associated with the agent $a$.

As a synthetic measure of trust for an agent $b$ in the category $\gamma, a$ considers the measure $\operatorname{trust}(b, \gamma)=W_{\text {rel }} \cdot \operatorname{rel}(b, \gamma)+(1-$ $\left.W_{r e l}\right) \cdot \operatorname{rep}(b, \gamma)$ where $W_{r e l}$ is a weight ranging in [0..1] representing the importance that $a$ assigns to the reliability with respect to the reputation.

\section{THE FRIENDSHIP AND GROUP FORMATION (FGF) ALGORITHM}

As described in Section II, for an agent $a$ is preferable to ask for a contribution or a recommendation a friend or a member of one of his groups, since in this case he will not pay any cost. The difference between a friend $f r$ and a group member $m$, from the viewpoint of $a$, is that $f r$ has a direct connection with $a$, in the sense that $a$ and $f r$ mutually accepted in the past to become friends. Instead, $m$ could not be a friend of $a$ and thus he is available to give contributions and recommendations to $a$ for free due to the fact they belong to the same group.

At each instant of time and for each category $\gamma$, the agent $a$ can compute a set of $\gamma$-preferable contributors $P C_{a}^{\gamma}$, containing the agents $b$ with which $a$ interacted in the past for obtaining a contribution related to the category $\gamma$, and having (i) the highest $c M a x$ trust values $\operatorname{trust}(b, \gamma)$ and (ii) a trust value greater than a fixed threshold $t c$. The agents belonging to $P C_{a}^{\gamma}$ are those that $a$ would prefer to contact to have a contribution. Analogously, the agent $a$ can compute a set of preferable recommenders $P R_{a}$, containing the agents $b$ with which $a$ interacted in the past for obtaining a recommendation, and having ( $i$ ) the highest $r$ Max honesty values hon $(b)$ and an honesty value greater than a fixed threshold $t h$. The agents belonging to $P R_{a}$ are those that $a$ would prefer to contact to have a recommendations.

In order to obtain the maximum performances, $a$ would desire to have in his set of friends $F_{a}$, or in some group $g \in G_{a}$ with which he is joined, only the agents belonging to the sets of $\gamma$-preferable contributors $P C_{a}^{\gamma}$ (for all the categories $\gamma$ ) and the set of preferable recommenders $P R_{a}$. In other words, he would desire to achieve the following goal:

$$
\bigcup_{\gamma \in C} P C_{a}^{\gamma} \bigcup P R_{a}=F_{a} \bigcup_{g \in G_{a}} g
$$

However, it is possible that $(i)$ some agents that do not belong to $\bigcup_{\gamma \in C} P C_{a}^{\gamma} \bigcup P R_{a}$ there exist in $F_{a} \bigcup_{g \in G_{a}} g$ and (ii) some agents that belong to $\bigcup_{\gamma \in C} P C_{a}^{\gamma} \bigcup P R_{a}$ are not present in $F_{a} \bigcup_{g \in G_{a}} g$. Both these situations imply a disadvantage for $a$, that must be available to satisfy for free possible requests coming from the agents that belongs to $F_{a} \bigcup_{g \in G_{a}} g$ without balancing this cost with the possibility to have in its turn the best interlocutors for obtaining contributions and/or recommendations.

In the situation (i) the disadvantage for $a$ can be represented by the percentage of the agents $b \in F_{a} \bigcup_{g \in G_{a}} g-$ $\bigcup_{\gamma \in C} P C_{a}^{\gamma} \cup P R_{a}$ (w.r.t. the number of agents present in $F_{a} \bigcup_{g \in G_{a}} g$ ), since each of these agents will not never contacted for requesting help while they can contact $a$ obtaining help for free.

In the situation (ii) instead, the disadvantage for $a$ can be represented computing, for each agent $b$ of $\bigcup_{\gamma \in C} P C_{a}^{\gamma}$ that is not present in $F_{a} \bigcup_{g \in G_{a}} g$, the difference between $\operatorname{trust}\left(b, \gamma^{*}\right)$ and $\operatorname{trust}\left(a l t_{b}, \gamma^{*}\right)$, where $\gamma^{*}$ is the category in which $b$ is one of the preferred agents (in case $b$ is the preferred agents in more categories, $\gamma^{*}$ is the category associated with the highest trust value) and $a l t_{b}$ is the best alternative to $b$ among the agents of $F_{a} \bigcup_{g \in G_{a}} g$, i.e. the agent of $F_{a} \bigcup_{g \in G_{a}} g$ having the best trust value in category $\gamma^{*}$. Analogously, it is necessary to cmpute for each agent $b$ of $\bigcup_{\gamma \in C} P R_{a}^{\gamma}$ that is not present in $F_{a} \bigcup_{g \in G_{a}} g$, the difference between hon $(b)$ and hon $\left(\right.$ alt $\left._{b}\right)$, where alt $_{b}$ is the best alternative to $b$ among the agents of $F_{a} \bigcup_{g \in G_{a}} g$. The whole disadvantage can be considered equal to the average of all these contributions.

In other word, at each time the disadvantage of $a$ will be expressed by this formula:

$$
D_{a}=\frac{P_{1}+P_{2}+P_{3}}{3}
$$

where:

$$
\begin{gathered}
P_{1}=\frac{\left\|F_{a} \bigcup_{g \in G_{a}} g-\bigcup_{\gamma \in C} P C_{a}^{\gamma} \bigcup P R_{a}\right\|}{\left\|F_{a} \bigcup_{g \in G_{a}} g\right\|} \\
P_{2}=\frac{\sum_{b \in \bigcup_{\gamma \in C} P C_{a}^{\gamma}-F_{a} \bigcup_{g \in G_{a}} g} \operatorname{trust}\left(b, \gamma^{*}\right)-\operatorname{trust}\left(\text { alt }_{b}, \gamma^{*}\right)}{\left\|\bigcup_{\gamma \in C} P C_{a}^{\gamma}-F_{a} \bigcup_{g \in G_{a} \|} g\right\|} \\
P_{3}=\frac{\sum_{b \in P R_{a}-F_{a} \bigcup_{g \in G_{a}} g} h o n(b)-h o n\left(\text { alt }_{b}\right)}{\left\|P R_{a}-F_{a} \bigcup_{g \in G_{a} \|} g\right\|}
\end{gathered}
$$

Obviously, if the equality 1 holds, the disadvantage $D_{a}$ will be equal to 0 (minimum value), while if $(i)$ all the agents belonging to $F_{a} \cup_{g \in G_{a}} g$ are not preferred contributors or recommender, and (ii) all the alternative agents introduce a trust difference equal to 1 , therefore the disadvantage $D_{a}$ will be equal to 1 (maximum value).

The algorithm we propose is executed by the agent $a$ for minimizing $D_{a}$ during several epochs, such that in each epoch some preferred agents are joined to the set $F_{a} \bigcup_{g \in G_{a}} g$ replacing those agents having the worst trust or honesty values. 
The period of time between two consecutive epochs is equal to a pre-fixed value $T$.

At each epoch, the algorithm is composed by the following two tasks, the former (called active task) dedicated to manage the requests that $a$ sends to the other agents, the second (called passive task) managing the requests coming from the other agents:

\section{A. Active task}

The agent $a$ executes this task in order to obtaining the friendship or the presence in a group of $G_{a}$ of those agents belonging to $\bigcup_{\gamma \in C} P C_{a}^{\gamma} \cup P R_{a}$ but which do not yet belong to $F_{a} \bigcup_{g \in G_{a}} g$. To this end, the strategy of $a$ is that of $(i)$ first requesting the friendship of each missing agent $b$; (ii) if the missing agent $b$ does not accept the friendship, then requesting to join with some group that contains $b$; (iii) if any group containing $b$ does not accept the joining request, then trying to forming a new group that in the future could attact $b$, requesting the participation of other agents having similar necessities.

More in particular, this task is composed by the following steps:

1) The set $\bigcup_{\gamma \in C} P C_{a}^{\gamma} \cup P R_{a}$ is computed.

2) For each agent $b \in \bigcup_{\gamma \in C} P C_{a}^{\gamma} \cup P R_{a}-F_{a} \bigcup_{g \in G_{a}} g$, a request of friendship is sent to $b$.

3) If the friendship request is accepted by $b$, then $b$ is added to $F_{a}$. Moreover, if $b \in P C_{a}^{\gamma}$, then the agent $k \in F_{a}$, $k \notin \bigcup_{\gamma \in C} P C_{a}^{\gamma} \cup P R_{a}$ having the worst trust value $\operatorname{trust}(k, \gamma)$ is removed from $F_{a}$. Otherwise, if $b \in P R_{a}$, the agent $k \in F_{a}, k \notin \bigcup_{\gamma \in C} P C_{a}^{\gamma} \cup P R_{a}$ having the worst honesty value $h o n(k)$ is removed from $F_{a}$.

4) If the friendship request is not accepted by $b$, then the agent $a$ requires to the $D F$ the set $G R O U P_{b}$ of all the groups containing $b$ as a member. For each group $g \in G R O U P_{b}$, the agent $a$ computes the disadvantage $D_{a}^{*}$ that would derive if the group $g$ is added to $G_{a}$, using the Formula 2. If $D_{a}^{*}<D_{a}$, then $a$ sends a joining request to $g$.

5) If the joining request is accepted by $g$, then $g$ is added to $G_{a}$. Moreover, analogously to the previous step, if $b \in$ $P C_{a}^{\gamma}$, then the agent $k \in F_{a}, k \notin \bigcup_{\gamma \in C} P C_{a}^{\gamma} \bigcup P R_{a}$ having the worst trust value $\operatorname{trust}(k, \gamma)$ is removed from $F_{a}$. Otherwise, if $b \in P R_{a}$, the agent $k \in F_{a}$, $k \notin \bigcup_{\gamma \in C} P C_{a}^{\gamma} \cup P R_{a}$ having the worst honesty value $h o n(k)$ is removed from $F_{a}$.

6) If the joining request is not accepted by $g$, then $a$ sends a call for a new group to all the agents belonging to $F_{a} \bigcup_{g \in G_{a}} g$.

7) When some agent affirmatively responses to the call for a new group, the new group is formed and registered to the DF.

\section{B. Passive task}

The purpose of this task is that of managing the requests of friendships coming from other agents, as well as the requests of joining that other agents send to groups with which $a$ is joined. In particular, this task is composed by the following steps:

1) When a friendship request coming from an agent $b$ arrives to $a$, then $a$ will accept it if the insertion of $b$ in the set $F_{a}$ (with the consequent removing of an agent following the rules described in step 3 of the active task) implies a decrement of the disadvantage $D_{a}$. Otherwise, the request will be refused.

2) When a joining request coming from an agent $b$ arrives to the administrator of a group $g$ with which the agent $a$ is joined, a voting is requested from the administrator to all the agents belonging to the group $g$. Each vote can be positive or negative. If the the majority of the votes is positive, then the joining request of $b$ is accepted, otherwise it is refused. The agent $a$ will give a positive vote if the insertion of $b$ in the set $F_{a}$ (with the consequent removing of an agent following the rules described in step 3 of the active task) implies a decrement of the disadvantage $D_{a}$. Otherwise, the vote of $a$ will be negative.

3) If a call for a new group arrives from an agent $b$, then $a$ accepts the proposal to join with the new group if the addition of $b$ to $F_{a} \bigcup_{g \in G_{a}} g$ does not increase the disadvantage $D_{a}$.

\section{TheORETICAL RESUlts}

We define Social Capital $S C$ of the whole multi-agent system the mean value of all the contributions $\left(1-D_{a}\right)$ given by each agent $a$. In other words, the social capital represents the average advantage associated to a given internal organization of the MAS in friendships and groups.

Definition 1: The Social Capital of a MAS is defined as:

$$
S C=\frac{\sum_{a \in A}\left(1-D_{a}\right)}{\|A\|}
$$

In this section, we provide two important results related to the FGF algorithm. First, we prove that at each iteration of the FGF, the social capital $S C$ increases. In other words, we show that FGF achieves the purpose of creating relationships among agents tending at optimizing the global social utility.

Theorem 1: The social capital $S C$ increases at each new iteration of the FGF algorithm.

Proof: At each iteration, each agent $a$ performs only actions that can either (i) increase each $D_{a}$, in the case some preferred contributor or recommender accept his joining request or (ii) maintain unvaried $D_{a}$, if any agent does not accept hos joining request. However, $D_{a}$ can decrease due to actions performed by other agents, as the cancellation of a preferred contributor or recommender from $F_{a}$ or from a group of $G_{a}$. However, let $b$ a preferred contributor in a category $\gamma^{*}$ or a recommender that cancels himself from $F_{a}$ or from a group of $G_{a}$. Then, the disadvantage $D_{a}$ will increase for the necessity to replace $b$ with the best alternative $a l t_{b}$, and the increment will be equal to $\operatorname{trust}\left(b, \gamma^{+}\right)-\operatorname{trust}\left(a_{1} t_{b}, \gamma^{*}\right)$ (or $h o n(b)-h o n\left(a l t_{b}\right)$ in the case of a recommender), that is lesser than 1. However, $b$ cancels himself from $F_{a}$ of 
from a group of $G_{a}$ only if $a$ is not one of his preferred contributors or recommenders, thus the cancellation implies a decrement of $D_{b}$ equal to 1 . Overall, the sum of $D_{a}$ and $D_{b}$ will decrement of $1-\left(\operatorname{trust}\left(b, \gamma^{*}\right)-\operatorname{trust}\left(\right.\right.$ alt $\left.\left._{b}, \gamma^{*}\right)\right)$ (or $1-\left(\operatorname{hon}(b)-\operatorname{hon}\left(\right.\right.$ alt $\left.\left._{b}\right)\right)$. From this observation, we directly derive that the sum of all the agent disadvantages decreases at each iteration, and consequently the sum of all the contributions $(1-D-a)$ increases. This proves the theorem.

In order to characterizing the global trustworthiness that an agent $a$ receives from the whole community in a given category $\gamma$, we define the notion of merit $\mu_{a}^{\gamma}$, as follows:

Definition 2: The merit $\mu_{a}^{\gamma}$ of an agent $a$ in the category $\gamma$ is defined as the number of agents that consider $a$ as a preferred contributor or a preferred recommender.

Moreover, we define also the notion of expected gain of an agent $a$, for characterizing how much the agent $a$ can expect to increment his bank amount at a given step of the competition. Denoting as $P_{a}(i)$ the probability distribution of the bank amount increment for $a$, i.e. the probability that the bank amount increment is equal to $i$, we have that:

Definition 3: The expected gain $\delta_{a}$ of the agent $a$ is defined as the expected value of the probability distribution $P_{a}(i)$

Finally, we consider valid the following mirror assumption:

Assumption 1: Let $a, b$ be two agents, such that at a given iteration $\mu_{a}^{\gamma}<\mu_{b}^{\gamma}$. In this situation the number $u_{a}$ of users contacting $a$ for a service request in the category $\gamma$ will be lesser than the number $u_{b}$ of users contacting $b$.

This assumption appears reasonable considering that if $\delta_{a}^{\gamma}<\delta_{b}^{\gamma}$, then the global satisfaction of the agent community for the performances of $a$ is lesser than the satisfaction for the performances of $b$. Since the global satisfaction of the agent community is constructed based on the feedbacks received by the users, it is reasonable to think that in this situation also the users will prefer to contact $a$ instead of contacting $b$. In other words, this assumption means that the users' choices specularly reflect the agents' choices. This will be particularly true if the trustworthiness of $a$, represented by the number of agents that consider $a$ as a preferred interlocutor, actually capture the expertise of $a$. Obviously, the more the trust models of the agents are built strictly based on the users' feedbacks, similarly to the case of the trust model presented in Section III, the more the mirror assumption can be considered as valid. Furthermore, the more the adopted trust model is able to capture the actual expertises of the agents, the more the assumption above will reflect the real situation.

Theorem 2: At each iteration, for each pair of agents $a, b$ such that $\mu_{a}^{\gamma}<\mu_{b}^{\gamma}$, the expected gain $\delta_{a}$ will be lesser than the expected gain $\delta_{b}$.

Proof: Let $a, b$ be two agents, such that at a given iteration $\mu_{a}^{\gamma}<\mu_{b}^{\gamma}$. Supposing as valid the mirror assumption, the number $u_{a}$ of users contacting $a$ for a service request related to $\gamma$ will be lesser than the number $u_{b}$ of users contacting $b$. Moreover, in this situation, the probability $P I_{a}$ that $a$ is contacted by other agents for a contribution or a recommendation related to $\gamma$ is lesser than the corresponding probability $P B_{a}$, and therefore the expected number of the agents $i_{a}$ contacting $a$ for a contribution or for a recommendation related to $\gamma$ is lesser than the expected number $i_{b}$ of those contacting $b$. In the same way, the expected number of agents $o_{a}$ contacted by $a$ in the category $\gamma$ is greater than the expected number of agents $o_{b}$ contacted by $b$, due to the high probability that the expertise of $a$ is smaller than that of $b$. Thus, also supposing for simplicity that both the price of a contribution or a recommendation is equal to $p^{*}$, the expected gain $\delta_{a}$ at the end of the current iteration is $u_{a} \cdot p+i_{a} \cdot p^{*}-o_{a} \cdot p^{*}$, that is smaller than the corresponding gain $\delta_{b}$ for the agent $b$.

\section{RELATED WORK}

This section discusses some previous work related to the issues of partner selection and collaboration among selfinterested agents. Partner selection plays an important role in filling the deficit of distributed agents. Research on partner selection generally proposes various types of evaluation metrics for selecting appropriate partners. Local decision with local modeling [12], [16] takes into account the local model about potential partners and finds the most appropriate partners, for example, according to trustworthiness, reputation or quality of provided services. These models are constructed either by direct observation or communication with other agents. In social control research, agents need to evaluate other agents or the services provided by other agents in order to realize a distributed but secure control over the interactions among agents [8].

The negotiation-based approach involves explicit peer-topeer communication for negotiation. The Contract Net Protocol (CNP) [21], [6] provides a mechanism for finding the best partners who provide necessary services at the least cost. The Adaptive Decision Making Framework (ADMF) [2] also deploys a negotiation-based partner selection scheme. Recently, the use of trust in competitive agent systems has been widely emphasized [17], [11]. In this context, trust measures have been exploited for forming clusters of agents [10], [3] and for generating recommendations in social network contexts [7]. The problem of detecting group of actors in a competitive social community based on trust has particularly been faced in [13], [18], [14]. None of the aforementioned approaches faces the issue of improving the social capital of the agent community by introducing meritocracy. Instead, those approaches try to use trust measures for recommending to an agent the best agents to contact as promising interlocutors, without the purpose of introducing a social advantage for the whole community. Instead, our approach is capable of achieving such an advantage, also realizing it through a meritocratic approach, that encourages the social actors to assume correct behaviours for increasing their social reputation.

\section{CONCLUSION}

The problem of introducing a convenient organization into a community of self-interested agent is central in the context of allowing agents to collaborate for increasing the individual capability of providing services to users. If in the past the 
most of the proposed approaches face such a problem trying to maximize the profits of the single actors or the whole community, however it is important to remark that these kinds of proposals lead to important negative effects as, for instance: (i) encouraging deceptive or fraudolent behaviours in the case the goal of the approach is that of rewarding the most aggressive agents or (ii) introducing a social flattening that does not take into account the different merits of individual agents, in the case the approach is focused on the optimization of the profit associated with the entire community. In this work, we argue that a possibility to avoid the above problems is that of using as objective function to maximize a measure of social capital, depending on the trust relationships existing among agents. In other words, in our vision the social capital of the community is represented by the strength of these trust relationships in the friendship lists and in the groups formed into the community. As a consequence, in this vision, those agents that are perceived as the most trustworthy are rewarded by our approach, thus introducing a form of meritocracy in the community. Our paper gives the theoretical demonstration that, at each iteration of the FGF algorithm (i) the social capital $S C$ increases and (ii) for each pair of agents $a, b$ such that $\mu_{a}^{\gamma}<\mu_{b}^{\gamma}$, the expected gain $\delta_{a}$ will be lesser than the expected gain $\delta_{b}$. This second result has been proved under the mirror assumption, valid in the case the behaviour of the users when selecting the agents reflects the behaviour of the agents when selecting their collaborators.

Our ongoing research is now devoted to test the FGF algorithm in complex situations, characterized by large sets of agents, and in real cases when agents operate on the behalf of human users. In particular, we are planning to develop an application in the domain of grid/cloud services, that appears as a promising possibility of profitably using our approach for introducing a convenient organization in the service system.

\section{REFERENCES}

[1] Agent uno: Winner in the 2nd Spanish ART Competition, author=Muoz, $\mathrm{V}$ and Murillo, J, journal=Inteligencia Artificial, volume $=12$, pages $=19$ 27, year $=2008$.

[2] K Suzanne Barber and Cheryl E Martin. Dynamic reorganization of decision-making groups. In Proceedings of the fifth international conference on Autonomous agents, pages 513-520. ACM, 2001.

[3] Francesco Buccafurri, Antonello Comi, Gianluca Lax, and Domenico Rosaci. A trust-based approach to clustering agents on the basis of their expertise. In Proceedings of Advances in Agent and Multi-Agent Systems 2014. AMSTA 2014, 2014.
[4] J Carbo, G Muller, M Gomez, and J Sabater-Mir. Improving the arttestbed, thoughts and reflections. In Proceedings of the Workshop on Competitive agents in "Agent Reputation and Trust Testbed", Salamanca, Spain, pages 1-15, 2008.

[5] Viet Dung Dang and Nicholas R Jennings. Generating coalition structures with finite bound from the optimal guarantees. In Proceedings of the Third International Joint Conference on Autonomous Agents and Multiagent Systems-Volume 2, pages 564-571. IEEE Computer Society, 2004.

[6] Randall Davis and Reid G Smith. Negotiation as a metaphor for distributed problem solving. Artificial intelligence, 20(1):63-109, 1983.

[7] Pasquale De Meo, Antonino De Meo, Domenico Rosaci, and Domenico Ursino. Recommendation of reliable users, social networks and highquality resources in a social internetworking system. AI Communications, 24(1):29-50, 2011.

[8] Keith Decker, Katia Sycara, and Mike Williamson. Middle-agents for the internet. In IJCAI (1), pages 578-583, 1997.

[9] Partha Sarathi Dutta and Sandip Sen. Forming stable partnerships. Cognitive Systems Research, 4(3):211-221, 2003.

[10] Salvatore Garruzzo and Domenico Rosaci. Agent clustering based on semantic negotiation. ACM Transactions on Autonomous and Adaptive Systems (TAAS), 3(2), 2008.

[11] Salvatore Garruzzo, Domenico Rosaci, and Giuseppe M.L. Sarné. Integrating trust measures in multi-agent systems. International Journal of Intelligent Systems (IJIS), 27(1):1-15, 2012.

[12] E Michael Maximilien and Munindar P Singh. Agent-based trust model involving multiple qualities. In Proceedings of the fourth international joint conference on Autonomous agents and multiagent systems, pages 519-526. ACM, 2005.

[13] Fabrizio Messina, Giuseppe Pappalardo, Domenico Rosaci, Corrado Santoro, and Giuseppe ML Sarné. A distributed agent-based approach for supporting group formation in $\mathrm{p} 2 \mathrm{p}$ e-learning. In Proceedings of the thirteenth International Conference on Advances in Artificial Intelligence. AI*IA 2013, 2013.

[14] Fabrizio Messina, Giuseppe Pappalardo, Domenico Rosaci, Corrado Santoro, and Giuseppe ML Sarné. Hyson: A distributed agent-based protocol for group formation in online social networks. In Proceedings of the 7th International Workshop on Multi-Agent Systems and Simulation (MATES 2013), 2013.

[15] M. Paldam. Social capital: one or many? Definition and measurement. Journal of Economic Surveys, 14(5):629-653, 2000.

[16] Jisun Park and K Suzanne Barber. Information quality assurance by lazy exploration of information source combinations space in open multiagent systems. J. UCS, 11(1):193-209, 2005.

[17] Domenico Rosaci. Trust measures for competitive agents. Knowledgebased Systems (KBS), 28(46):38-46, 2012

[18] Domenico Rosaci and Giuseppe ML Sarné. Matching users with groups in social networks. In Proceedings of the International Conference on Distributed Computing (IDC 2013), 2013.

[19] Tuomas Sandholm, Kate Larson, Martin Andersson, Onn Shehory, and Fernando Tohmé. Anytime coalition structure generation with worst case guarantees. arXiv preprint cs/9810005, 1998.

[20] W. Sherchan, S. Nepal, and C. Paris. A survey of trust in social networks. ACM Computing Surveys, 45(4):47, 2013.

[21] Reid G Smith and Randall Davis. Frameworks for cooperation in distributed problem solving. Systems, Man and Cybernetics, IEEE Transactions on, 11(1):61-70, 1981. 